\title{
The spectrum of thyroid disease and risk of new onset atrial fibrillation: a large population cohort study
}

\author{
(c) $(1)(9)$ OPEN ACCESS
}

\author{
Christian Selmer research fellow ${ }^{1}$, Jonas Bjerring Olesen research fellow ${ }^{1}$, Morten Lock Hansen \\ research fellow ${ }^{1}$, Jesper Lindhardsen research fellow ${ }^{1}$, Anne-Marie Schjerning Olsen research \\ fellow ${ }^{1}$, Jesper Clausager Madsen research fellow ${ }^{2}$, Jens Faber professor ${ }^{34}$, Peter Riis Hansen \\ research director ${ }^{1}$, Ole Dyg Pedersen research fellow ${ }^{5}$, Christian Torp-Pedersen professor ${ }^{14}$, \\ Gunnar Hilmar Gislason research director ${ }^{1}$
}

${ }^{1}$ Department of Cardiology, Gentofte University Hospital, Hellerup, Denmark; ${ }^{2}$ Copenhagen General Practitioners Laboratory, Copenhagen, Denmark; ${ }^{3}$ Department of Endocrinology, Herlev University Hospital, Herlev, Denmark; ${ }^{4}$ Faculty of Health Sciences, University of Copenhagen, Denmark; ${ }^{5}$ Department of Cardiology, Roskilde University Hospital, Copenhagen, Denmark

\begin{abstract}
Objectives To examine the risk of atrial fibrillation in relation to the whole spectrum of thyroid function in a large cohort of patients.

Design Population based cohort study of general practice patients identified by linkage of nationwide registries at the individual level. Setting Primary care patients in the city of Copenhagen.

Subjects Registry data for 586460 adults who had their thyroid function evaluated for the first time by their general practitioner during 2000-10 and who were without previously recorded thyroid disease or atrial fibrillation.
\end{abstract}

Main outcome measure Poisson regression models used to estimate risk of atrial fibrillation by thyroid function.

Results Of the 586460 individuals in the study population (mean (SD) age 50.2 (16.9) years, 39\% men), 562461 (96.0\%) were euthyroid, 1670 $(0.3 \%)$ had overt hypothyroidism, 12087 (2.0\%) had subclinical hypothyroidism, 3966 (0.7\%) had overt hyperthyroidism, and 6276 (1.0\%) had subclinical hyperthyroidism. Compared with the euthyroid individuals, the risk of atrial fibrillation increased with decreasing levels of thyroid stimulating hormone (TSH) from high normal euthyroidism (incidence rate ratio $1.12(95 \% \mathrm{Cl} 1.03$ to 1.21$))$ to subclinical hyperthyroidism with reduced TSH (1.16 (0.99 to 1.36)) and subclinical hyperthyroidism with supressed TSH (1.41 (1.25 to 1.59)). Both overt and subclinical hypothyroidism were associated with a lower risk of atrial fibrillation.

Conclusion The risk of atrial fibrillation was closely associated with thyroid activity, with a low risk in overt hypothyroidism, high risk in hyperthyroidism, and a TSH level dependent association with risk of atrial fibrillation across the spectrum of subclinical thyroid disease.

\section{Introduction}

Atrial fibrillation is a common cardiac arrhythmia and an important risk factor for ischaemic stroke and heart failure. Thyroid hormones have substantial effects on the cardiovascular system, and it is well known that overt hyperthyroidism is associated with atrial fibrillation. Subclinical hyperthyroidism (that is, reduced serum thyroid stimulating hormone (TSH) concentration but free thyroxine levels within reference ranges) is associated with elevated resting pulse, increased frequency of atrial and ventricular premature beats, increased left ventricular mass index, and cardiac output. ${ }^{12}$ Previous studies of subclinical hyperthyroidism have suggested increased risk of developing atrial fibrillation, but these studies were all performed in relatively small cohorts or using pooled data from several small cohorts. ${ }^{3-7}$ Also, because of the small sample sizes, it has been difficult to characterise subgroups of patients with subclinical hyperthyroidism in order to assess a possible "dose dependent" association between serum TSH concentration and risk of atrial fibrillation, sex differences, and the possible role of high normal thyroid function (low TSH within the normal reference range). How subclinical hyperthyroidism should be managed has therefore also been much debated. ${ }^{8}$

Overt hypothyroidism, however, is associated with bradycardia, ${ }^{9}$ dyslipidaemia, hypertension, atherosclerosis, decreased variability in heart rate, and increased risk of myocardial 
infarction. ${ }^{10}$ Imaging studies of patients with subclinical hypothyroidism (that is, with increased TSH, but free thyroxine within reference range) have shown subtle changes in cardiac performance. ${ }^{11}$ Despite these observations, neither overt nor subclinical hypothyroidism has been associated with increased or decreased risk of atrial fibrillation.

The prevalence of subclinical thyroid dysfunction is high in the adult population, ${ }^{12}$ and symptoms of thyroid disease are often mild - and, therefore, thyroid functions tests are often performed routinely in primary care on vague clinical indications.

Furthermore, thyroid testing is typically included in many standard laboratory test packages. This policy makes available large population based databases with thyroid test results. Patients followed in primary care are often more healthy than hospital patients (inpatients and outpatients) and therefore provide a unique possibility to assess the isolated effect of thyroid dysfunction on the development of atrial fibrillation. The purpose of the present study was to assess the relation between thyroid function and risk of atrial fibrillation in a large cohort of primary care patients.

\section{Methods}

\section{Study setting}

In Denmark, each resident is provided with a permanent and unique civil registration number, enabling individual level linkage between nationwide administrative registers holding information on healthcare use. ${ }^{13}$ Since 1978 the Danish National Patient Registry has registered all hospital contacts in Denmark. ${ }^{14}$ Each admission is registered with one primary diagnosis and, if appropriate, one or more secondary diagnoses based on the World Health Organization's ICD-10 (international classification of diseases, 10th revision).

The Danish Register of Medicinal Product Statistics holds information regarding all claimed prescriptions (coded according to the international Anatomical Therapeutic Chemical classification ${ }^{15}$ ) in Denmark since 1995. The registry also includes information on the date of drug dispensation, strength, and quantity dispensed. Because of partial reimbursement of drug expenses by the Danish healthcare authorities, all pharmacies are required to provide information that ensures complete and accurate registration. This registry has been found to be accurate and has been described in more detail previously. ${ }^{16}$

Vital status was obtained from the Central Population Register, which records all deaths within 14 days. ${ }^{17}$ Annual incomes for all Danish citizens were retrieved from the Integral Database for the Danish Labour Market, ${ }^{18}$ and socioeconomic status was defined by the average yearly gross household income in a five year period before inclusion in the study.

\section{Population}

The study cohort comprised citizens of Copenhagen (the capital of Denmark) aged 18 years or more who underwent thyroid screening at the Copenhagen General Practitioners Laboratory in the period of 1 January 2000 to 22 January 2010. Each subject entered the cohort on first thyroid screening and was followed until 31 December 2010, migration from the study area, or death. The Copenhagen General Practitioners Laboratory handles all laboratory tests ordered by primary care physicians in the Copenhagen area except for the municipality of Frederiksberg. $\mathrm{TSH}$, free thyroxine, and total thyroxine were determined in serum by the commercially available ADVIA Centaur TSH kit (Bayer/Siemens, Tarrytown, NY) according to the instructions of the manufacturer. We excluded all subjects with a previous diagnosis of atrial fibrillation, thyroid dysfunction (that is, previous prescription of levothyroxine or antithyroid drugs or any thyroid related diagnosis), or previous use of amiodarone, vitamin $\mathrm{K}$ antagonists, and digoxin. Patients with ambiguous times of death or thyroid blood test values were also excluded.

Patients where categorised according to their thyroid status at the time of first blood sampling based on both the traditional definitions of thyroid dysfunction and using definitions based on TSH level for high normal euthyroidism and two levels of subclinical hyperthyroidism (table $1 \Downarrow$ ).

\section{Comorbidity and concomitant medical therapy}

From the Danish National Patient Registry we identified comorbidities, such as myocardial infarction, congestive heart failure, ischaemic stroke, etc (see appendix A on bmj.com). These diagnoses have been validated and have high sensitivity and positive predictive value. ${ }^{19}{ }^{20} \mathrm{We}$ calculated the Charlson comorbidity index on the basis of pre-specified diagnoses up to one year before entry to the study cohort. ${ }^{212}$ From the Danish Register of Medicinal Product Statistics we identified all claimed prescriptions of amiodarone and antithyroid medications including methimazole and propylthiouracil.

\section{Outcome}

The primary outcome of interest was a hospital diagnosis of new onset atrial fibrillation (ICD-10 code I48) as primary or secondary diagnosis.

\section{Statistical analysis}

Baseline characteristics are presented as numbers with percentages for categorical variables and as means (standard deviations) for continuous variables. Incidence rates were calculated as number of events per 1000 person years stratified by thyroid function. Cumulative incidence proportion curves were constructed for each thyroid condition in the age group $>65$ years, but not in the younger group because of few events before age 65 . Cumulative incidence was assessed using a competing risk model to account for the competing risk of death from all causes in the population. ${ }^{23}{ }^{24} \mathrm{We}$ considered the competing risk of all cause death in the analysis, because in the standard Kaplain-Meier approach people censored are assumed to continue to have the same risk as those still left in the study, and the analysis would artificially describe the risk of atrial fibrillation in a cohort of patients who cannot die. In the analysis provided, the cumulative incidence describes the risk of atrial fibrillation and still being alive.

Time dependent Poisson regression models were constructed to estimate incidence rate ratios (with $95 \%$ confidence intervals) for atrial fibrillation of new onset. The Poisson regression models were adjusted for age, sex, calendar year, Charlson comorbidity index, and socioeconomic status. Age and calendar year were included as time dependent variables. Socioeconomic status and Charlson comorbidity index were included as fixed categorical variables obtained at baseline. Follow-up was started after a 90 day grace period to avoid the risk of confounding by indication. Individuals were censored at the end of the follow-up period (31 December 2010), migration, or death. A 5\% significance level was used in all analyses, including when testing for interactions. All analyses were stratified by sex and age groups because of significant interaction with thyroid function.

Time dependent variables have been used because some important covariates are not necessarily constant through the whole study. For instance, age is one of the most important 
confounders, and is naturally a function of the calendar year and needs to be updated during follow-up to allow patients to contribute with person years in different age groups. Also, in our sensitivity analysis different treatment regimens are included as time dependent variables, allowing patients to contribute to person years to both treated and untreated groups. This was done by splitting each study subject into several observations, one for each status defined by the time dependent variable.

Several sensitivity analyses were performed. Firstly, we included any thyroid treatment (medication or thyroid related hospital diagnosis) as a time dependent variable, allowing the patients to change from an untreated state to a treated state over time. Secondly, we did the same sensitivity analysis using only methimazole and levothyroxine treatments as time dependent variables. Thirdly, we did a sensitivity analysis on the first 12 months after inclusion by splitting the follow-up time into three, six, and 12 months' follow-up, allowing individual subjects to contribute with person-years in all follow-up groups. Fourthly, we did a sensitivity analysis adjusting the main model for known risk factors of atrial fibrillation such as hypertension, heart failure, myocardial infarction, valvular heart disease, and diabetes defined at baseline. ${ }^{25}$ Fifthly, we included thyroid status as a time dependent variable during follow-up based on the individual laboratory analysis in the period. This sensitivity analysis allowed patients to change between thyroid status groups (table $1 \Downarrow$ ) at any time during follow-up, hence, patients only contribute with risk time in the thyroid status group they were in at any specific point in time. Finally, we did an analysis using a Charlson comorbidity index calculated on basis of diagnoses up to five years before cohort entry.

All statistical analyses were performed with the SAS Statistical Software version 9.2 (SAS Institute, Gary NC, USA) and Stata Software version 11 (StataCorp, College Station TX, USA).

\section{Results}

We identified a total of 586460 individuals from 2000-10 who had their thyroid function evaluated for the first time by their general practitioner and who were without previously recorded thyroid disease or atrial fibrillation. Figure $1 \Downarrow$ shows the selection of the study cohort. Table $2 \Downarrow$ shows baseline characteristics of the euthyroid patients and the groups of patients with thyroid dysfunction. Women predominated (61\%) in the study cohort and where slightly younger (about 2 years) than the men. The cohort had low degree of comorbidity, and there was a uniform distribution of socioeconomic status as estimated by yearly income. On average the patients with thyroid dysfunction were older and more commonly women. The group of patients with subclinical hyperthyroidism were significantly older than individuals in the other groups.

\section{Incidence of atrial fibrillation}

During the follow-up of 3215807 person years (median follow-up time 5.5 years), a total of 17154 (2.9\%) patients had a hospital diagnosis of first time atrial fibrillation, of whom $53 \%$ were women. Of these hospital diagnoses, in 52\% the atrial fibrillation was the primary reason for hospitalisation. Among the euthyroid subjects, $2.9 \%$ developed atrial fibrillation, in contrast to $4.6 \%$ of patients with hyperthyroidism and $2.5 \%$ with hypothyroidism. Table $3 \Downarrow$ shows the unadjusted and age adjusted incidence rates for atrial fibrillation stratified by age and sex.

Overall, age adjusted incidence rates for atrial fibrillation were lower in patients with overt hypothyroidism and higher in patients with overt hyperthyroidism compared with the euthyroid cohort. Patients with subclinical hypothyroidism had incidence rates similar to those of the euthyroid cohort, whereas the subclinical hyperthyroid patients had markedly higher rates of atrial fibrillation. Patients with overt hyperthyroidism had higher age adjusted rates of atrial fibrillation than those with subclinical hyperthyroidism. Figure $2 \Downarrow$ shows the cumulative incidence of atrial fibrillation for patients aged $>65$ years. Of 122351 individuals included in the competing risk calculations, 11879 (10\%) had an event of atrial fibrillation, 42213 (34\%) died, and $68259(56 \%)$ were censored because of migration or end of the study period.

Age adjusted rates of atrial fibrillation for levels of high normal thyroid function and two levels of subclinical hyperthyroidism showed a uniform development from low rates in euthyroid subjects to higher rates in subclinical hyperthyroidism with suppressed TSH (table $4 \Downarrow$ ). Figure $3 \Downarrow$ shows unadjusted incidence rates and adjusted incidence rate ratios.

\section{Poisson regression analysis}

Overall, an almost linear relation was seen between thyroid function and risk of atrial fibrillation (table $3 \Downarrow$ ) —-that is, from a decreased risk in overt hypothyroidism (incidence rate ratio 0.67 (95\% confidence interval 0.50 to 0.92$)$ ) and subclinical hypothyroidism $(0.87$ ( 0.79 to 0.97$))$ to an increased risk in subclinical hyperthyroidism (1.31 (1.19 to 1.44$))$ and overt hyperthyroidism (1.42 (1.22 to 1.63$)$ ) compared with the euthyroid state. This relation was observed in all examined subgroups except from the group of men aged $>65$ years with overt hyperthyroidism. The highest relative risk of atrial fibrillation was seen among young men with overt hyperthyroidism and young women with subclinical hyperthyroidism.

A similar almost linear relation was seen between mild hyperthyroid states based on TSH level (high normal euthyroid to subclinical hyperthyroid) and risk of atrial fibrillation (table $4 \Downarrow$, fig $3 \Downarrow)$.

\section{Supplementary sensitivity analysis}

The results from the sensitivity analyses are presented in appendix B on bmj.com. When including any treatment as a time dependent variable, we found a slight reduction in risk of atrial fibrillation in overt and subclinical hyperthyroidism, whereas the results for the hypothyroid states showed a slight accentuation of the risk reducing effect. Including methimazole and levothyroxine as time dependent variables also gave a slight risk reduction among the hyperthyroid patients, but it did not change the results significantly for the hypothyroid patients. The stratified sensitivity analysis at three, six, and 12 months of follow-up showed that overt hypothyroid patients had no difference in the risk for the three periods. The subclinical hypothyroid patients were normalised after 12 months. In overt hyperthyroid patients the risk of atrial fibrillation was markedly elevated in the first six months and then fell to a level comparable with that of the main model. The subclinical hyperthyroid patients showed a drop in risk of atrial fibrillation after six months and then an elevated risk after 12 months of follow-up.

Adjusting the main model for known risk factors of atrial fibrillation such as hypertension, heart failure, myocardial infarction, valvular heart disease, and diabetes did not alter the main results. Including thyroid status as a time dependent variable accentuated the risk estimates for the hyperthyroid states, and showed the same trend for overt hypothyroid subjects, whereas the results for the subclinical hypothyroid group became 
non-significant. Using the five year Charlson comorbidity index in the analysis did not alter the results.

\section{Discussion}

The current study is, to the best of our knowledge, the first to assess the association between the whole spectrum of thyroid disease and the subsequent risk of atrial fibrillation in a population of primary care patients. Our main finding was an apparent linear relation between levels of thyroid dysfunction and atrial fibrillation risk - that is, a low atrial fibrillation risk in hypothyroid patients, a high risk in hyperthyroidism, and a TSH level dependent (a dose-response relation) increased risk of atrial fibrillation in all levels of hyperthyroid disease, even in high normal euthyroid subjects. Notably, in subjects with reduced serum TSH levels but normal free thyroid hormone levels the risk of developing atrial fibrillation was increased approximately $10 \%$ in individuals with high normal thyroid function and increased about $40 \%$ in those with subclinical hyperthyroidism with suppressed TSH levels (table $4 \Downarrow$, fig $3 \Downarrow$ ). Overall, the relative risk of atrial fibrillation associated with thyroid dysfunction was highest among the younger subjects and lower in elderly subjects. This is because less of the atrial fibrillation risk is attributable to a hyperthyroid state in elderly people, whereas hyperthyroidism in younger adults is often the main cause of atrial fibrillation. This observation may be in line with the fact that the marked rise in atrial fibrillation incidence with increasing age is mainly due to increased cardiovascular comorbidity (such as hypertension, heart failure, myocardial infarction, valvular heart disease, and diabetes).

The eight year cumulative incidence of atrial fibrillation among our elderly subjects ( $>65$ years old) was approximately $13 \%$ in those with overt hyperthyroidism and slightly lower in those with subclinical hyperthyroidism (fig $2 \Downarrow$ ). This is more than $10 \%$ lower than previously reported by Sawin et $\mathrm{al}^{6}$ and $25 \%$ lower than reported by Cappola et $\mathrm{al}^{7}$ : it might be explained by increased focus on thyroid disease and cardiovascular risk in primary care, or the fact that these studies did not account for the effect of competing risk of death. Also, these previous studies showed no relationship between hypothyroidism and atrial fibrillation. Notably, we found a reduction in the cumulative incidence and overall risk of atrial fibrillation among hypothyroid subjects. One explanation for this finding may be that we studied a cohort with little comorbidity and, therefore, might observe a more direct physiological effect of the hypothyroid state. Another possibility is that hypothyroid patients are treated with levothyroxine, which is known to somewhat reverse cardiovascular risk factors (for example, thyromimetic drugs may decrease levels of atherogenic lipoproteins). ${ }^{26}$ Interestingly, the sensitivity analysis of overt hypothyroid patients showed a stable risk of atrial fibrillation during the first 12 months, whereas the subclinical hypothyroid subjects had no reduced risk of atrial fibrillation after 12 months. In the sensitivity analysis we assumed that most patients would be well treated during the first 12 months, and our results, although not significant, indicated that the level of hypothyroidism was inversely correlated to the risk of atrial fibrillation and that treatment normalised this relationship.

A previous cross sectional study including 5860 subjects $^{4}$ showed a relation between electrocardiographic evidence of atrial fibrillation and concentrations of free thyroxine (but not TSH levels) even in euthyroid subjects, suggesting that high normal thyroid function is indeed a risk factor for atrial fibrillation, as we found in the current study. A recent prospective cohort study by Heeringa et al of 1426 subjects showed that in euthyroid subjects there was a relation between risk of atrial fibrillation and levels of TSH and free thyroxine. ${ }^{5}$ A recent study by Collet et al, based on 10 prospective cohorts and a total of 52674 pooled participants, found an overall $68 \%$ increased risk of atrial fibrillation in subclinical hyperthyroid patients and a $150 \%$ increased risk in those with TSH levels $<0.1 \mathrm{mIU} / \mathrm{L} .{ }^{27}$ However, the latter finding was based on only four events; in comparison, we had 280 events in the same group and found only a $40 \%$ increased risk of atrial fibrillation.

Part of the results seen in prospective cohort studies could be explained by the fact that TSH levels even within the normal reference range predict future hypothyroidism and hyperthyroidism. ${ }^{28}$ However, our results and those of recent studies ${ }^{4}$ support the hypothesis that even subclinical hyperthyroidism and high normal euthyroid states increase the risk of atrial fibrillation. These results add to the ongoing discussion of whether the lower reference range of plasma TSH levels should be reconsidered. ${ }^{29}$

To our knowledge we are the first to demonstrate a protective effect of hypothyroidism towards atrial fibrillation. None of the above mentioned studies ${ }^{4}{ }^{28}$ were able to show such a relationship. Elevated TSH levels have previously been associated with increased longevity in both animal models ${ }^{30}$ and human studies, ${ }^{31}$ and an intriguing possibility is that the lower resting heart rate associated with hypothyroidism not only protects against atrial fibrillation but could also lower cardiovascular mortality. ${ }^{32}$

Most of the patients in this cohort from primary care will be treated, as appropriate, with radioiodine, antithyroid medication, or thyroid replacement therapy according to established clinical practice. Antithyroid treatment reverses the acute risk of atrial fibrillation in hyperthyroidism, ${ }^{33}$ and our results therefore suggest that much of the pathophysiological background for atrial fibrillation risk in the study was established in the period preceding thyroid screening - that is, a long term affection of the heart caused by the subclinical thyroid dysfunction. The results from the sensitivity analysis support this notion as the subjects with overt hyperthyroidism had a markedly increased risk of atrial fibrillation the first six months, which then fell to a level similar to the risk found in the main analysis. Interestingly, the risk of atrial fibrillation in subclinical hyperthyroidism was significantly increased after 12 months. This further underlines the importance of early detection and treatment of subclinical thyroid disease to avoid development of irreversible cardiovascular abnormalities as well as psychiatric and cognitive sequelae. ${ }^{34-36}$

Our results support the growing evidence that subclinical hyperthyroid and possibly also high normal euthyroid states are risk factors for atrial fibrillation and suggest that patients with these conditions should routinely be screened for atrial fibrillation. Whether more aggressive thyroid screening and treatment can reduce the risk of atrial fibrillation and its complications require further study.

\section{Strengths and limitations of study}

The main strength of this study was the large cohort of unselected subjects from primary care who had thyroid screening performed. However, as it was an observational study, it is not possible to draw direct conclusions on causal relationships of the findings. Specifically, it is impossible with the available data to explore the reasons for each individual subject undergoing thyroid screening. One can speculate that subjects who had a known thyroid problem would be more prone subsequently to have electrocardiograms taken (implicating 
surveillance bias). However, this does not explain the "dose-response" relationship we found between TSH levels and risk of atrial fibrillation.

The study was based on administrative registers that did not include clinical parameters correlated to outcome, such as body mass index, smoking status, serum lipid levels, thyroid autoantibody levels, and echocardiographic or electrocardiographic findings. Neither did we have data on the types of hyperthyroidism, such as Graves' disease or nodular disease. $^{37}$

Patients were categorised by only their first time thyroid screening, and we did not take into account the possibility that subjects in the euthyroid group might develop thyroid dysfunction during follow-up and subsequently atrial fibrillation or that possible undertreatment or overtreatment of patients with thyroid dysfunction might alter their risk of atrial fibrillation due to changes in TSH and free thyroxine levels. However, a sensitivity analysis with time dependent adjustment for treatment defined as any thyroid related diagnosis or thyroid related medication did not alter the results significantly.

Also, we were unable to detect patients with new onset atrial fibrillation not diagnosed at a hospital who were treated by their general practitioner nor patients with asymptomatic paroxysmal atrial fibrillation, which could lead to detection bias. However, most patients diagnosed with atrial fibrillation in Denmark have a hospital admission or contact with an outpatient hospital clinic and the atrial fibrillation diagnosis in the Danish registers has previously been validated and has a sensitivity of $88 \%$ and a specificity of $88-99 \%{ }^{38-40}$ Furthermore, the possibility of asymptomatic paroxysmal atrial fibrillation in the cohort would probably be non-differential or bias towards the null hypothesis.

The Danish population comprises mainly white people, and so extrapolation of these results to other ethnic groups should be done with care.

\section{Conclusions}

In a population based sample of primary care patients who were subjected to thyroid function blood tests, we found a TSH level dependent relation between the degree of thyroid dysfunction and risk of new onset atrial fibrillation. Our results showed that even discrete subclinical hyperthyroidism was associated with increased risk of atrial fibrillation. Notably, both subclinical and overt hypothyroidism were associated with lower risk of atrial fibrillation. These results support long term screening for atrial fibrillation in patients with thyroid disease.

Contributors: CS made primary contributions to data collection and analysis, interpretation of results, and writing the manuscript. GHG and ODP helped to write the first draft. JBO, GHG, MLH, ODP, JL, and CT-P contributed to the study conception and design. All authors contributed to interpretation of results, all revised the manuscript critically for important intellectual content, and all approved the final manuscript. CS is the guarantor.

Funding: The study was partly funded by unrestricted grants from the Danish Heart Foundation (No 11-04-R84-A3483-22669), Danish Thyroid Association, Agnes and Knut Mørk Foundation, and FUKAP and START Foundations, Gentofte University Hospital, Denmark.

Competing interests: All authors have completed the ICMJE uniform disclosure form at www.icmje.org/coi_disclosure.pdf (available on request from the corresponding author) and declare: no support from any organisation for the submitted work; no financial relationships with any organisations that might have an interest in the submitted work in the previous three years; no other relationships or activities that could appear to have influenced the submitted work.
Ethical approval: Retrospective register studies do not require ethical approval in Denmark. This study was approved by the Danish Data Protection Agency (reference 2007-41-1667). All authors had full access to the data and take full responsibility for their integrity.

Data sharing: No additional data available.

1 Biondi B, Palmieri EA, Fazio S, Cosco C, Nocera M, Saccà L, et al. Endogenous subclinical hyperthyroidism affects quality of life and cardiac morphology and function in young and middle-aged patients. J Clin Endocrinol Metab 2000;85:4701-05.

2 Faber J, Wiinberg N, Schifter S, Mehlsen J. Haemodynamic changes following treatment of subclinical and overt hyperthyroidism. Eur J Endocrinol 2001;145:391-6.

3 Collet TH, Gussekloo J, Bauer DC, den Elzen WP, Cappola AR, Balmer P, et al. Subclinical hyperthyroidism and the risk of coronary heart disease and mortality. Arch Intern Med 2012;172:799-809.

4 Gammage MD, Parle JV, Holder RL, Roberts LM, Hobbs FD, Wilson S, et al. Association between serum free thyroxine concentration and atrial fibrillation. Arch Intern Med 2007:167:928-34.

5 Heeringa J, Hoogendoorn EH, van der Deure WM, Hofman A, Peeters RP, Hop WC, et al. High-normal thyroid function and risk of atrial fibrillation: the Rotterdam study. Arch Intern Med 2008;168:2219-24.

6 Sawin CT, Geller A, Wolf PA, Belanger AJ, Baker E, Bacharach P, et al. Low serum thyrotropin concentrations as a risk factor for atrial fibrillation in older persons. $N$ Engl $J$ Med 1994;331:1249-52.

7 Cappola AR, Fried LP, Arnold AM, Danese MD, Kuller LH, Burke GL, et al. Thyroid status, cardiovascular risk, and mortality in older adults. JAMA 2006;295:1033-41.

8 Parle JV, Franklyn JA, Cross KW, Jones SC, Sheppard MC. Prevalence and follow-up of abnormal thyrotrophin (TSH) concentrations in the elderly in the United Kingdom. Clin Endocrinol 1991;34:77-83.

9 Klein I, Ojamaa K. Thyroid hormone and the cardiovascular system. N Engl J Med 2001;344:501-9.

10 Kahaly GJ. Cardiovascular and atherogenic aspects of subclinical hypothyroidism. Thyroid 2000;10:665-79

11 Fazio S, Biondi B, Carella C, Sabatini D, Cittadini A, Panza N, et al. Diastolic dysfunction in patients on thyroid-stimulating hormone suppressive therapy with levothyroxine: beneficial effect of beta-blockade. J Clin Endocrinol Metab 1995;80:2222-6.

12 Hollowell JG, Staehling NW, Flanders WD, Hannon WH, Gunter EW, Spencer CA, et al. Serum TSH, T(4), and thyroid antibodies in the United States population (1988 to 1994): National Health and Nutrition Examination Survey (NHANES III). J Clin Endocrinol Metab 2002;87:489-99.

13 Pedersen CB. The Danish Civil Registration System. Scand J Public Health 2011;39(7 suppl):22-5.

14 Lynge E, Sandegaard JL, Rebolj M. The Danish National Patient Register. Scand J Public Health 2011;39(7 suppl):30-3.

15 WHO Collaborating Centre for Drug Statistics Methodology. Guidelines for ATC classification and DDD assignment, 2012.www.whocc.no/atc_ddd_publications/guidelines/

16 Kildemoes HW, Sørensen HT, Hallas J. The Danish National Prescription Registry. Scand $J$ Public Health 2011:39(7 suppl):38-41.

17 Helweg-Larsen K. The Danish Register of Causes of Death. Scand J Public Health 2011;39(7 suppl):26-9.

18 Baadsgaard M, Quitzau J. Danish registers on personal income and transfer payments. Scand J Public Health 2011;39(7 suppl):103-5.

19 Madsen M, Davidsen M, Rasmussen S, Abildstrom SZ, Osler M. The validity of the diagnosis of acute myocardial infarction in routine statistics: a comparison of mortality and hospital discharge data with the Danish MONICA registry. J Clin Epidemiol and hospital dischar:56:124-30.

20 Krarup LH, Boysen G, Janjua H, Prescott E, Truelsen T. Validity of stroke diagnoses in a National Register of Patients. Neuroepidemiology 2007;28:150-4.

21 Nuttall M, van der Meulen J, Emberton M. Charlson scores based on ICD-10 administrative data were valid in assessing comorbidity in patients undergoing urological cancer surgery. $\checkmark$ Clin Epidemiol 2006;59:265-73.

22 Thygesen SK, Christiansen CF, Christensen S, Lash TL, Sørensen HT. The predictive value of ICD-10 diagnostic coding used to assess Charlson comorbidity index conditions in the population-based Danish National Registry of Patients. BMC Med Res Methodol 2011;11:83.

23 Fine JP, Gray RJ. A proportional hazards model for the subdistribution of a competing risk. J Am Stat Assoc 1999

24 Satagopan JM, Ben-Porat L, Berwick M, Robson M, Kutler D, Auerbach AD. A note on competing risks in survival data analysis. Br J Cancer 2004;91:1229-35.

25 Benjamin EJ, Levy D, Vaziri SM, D'Agostino RB, Belanger AJ, Wolf PA. Independent risk factors for atrial fibrillation in a population-based cohort. The Framingham Heart Study. JAMA 1994:271:840-4.

26 Ladenson PW, Kristensen JD, Ridgway EC, Olsson AG, Carlsson B, Klein I, et al. Use of the thyroid hormone analogue eprotirome in statin-treated dyslipidemia. N Engl J Med 2010;362:906-16.

27 Collet TH, Gussekloo J, Bauer DC, den Elzen WP, Cappola AR, Balmer P, et al. Subclinical hyperthyroidism and the risk of coronary heart disease and mortality. Arch Intern Med 2012;172:799-809.

28 Asvold BO, Vatten LJ, Midthjell K, Bjøro T. Serum TSH within the reference range as a predictor of future hypothyroidism and hyperthyroidism: 11-year follow-up of the HUNT Study in Norway. J Clin Endocrinol Metab 2012;97:93-9.

29 Goichot B, Sapin R, Schlienger JL. Subclinical hyperthyroidism: considerations in defining the lower limit of the thyrotropin reference interval. Clin Chem 2009;55:420-4.

30 Buffenstein R, Pinto M. Endocrine function in naturally long-living small mammals. Mol Cell Endocrinol 2009;299:101-11.

31 Atzmon G, Barzilai N, Hollowell JG, Surks MI, Gabriely I. Extreme longevity is associated with increased serum thyrotropin. J Clin Endocrinol Metab 2009;94:1251-4.

32 Jensen MT, Marott JL, Allin KH, Nordestgaard BG, Jensen GB. Resting heart rate is associated with cardiovascular and all-cause mortality after adjusting for inflammatory markers: the Copenhagen City Heart Study. Eur J Prev Cardiol 2012;19:102-8.

33 Biondi B, Palmieri EA, Lombardi G, Fazio S. Effects of subclinical thyroid dysfunction on the heart. Ann Intern Med 2002;137:904-14. 


\section{What is already known on this topic}

Patients with overt hyperthyroidism have increased risk of atrial fibrillation

Information on the risk of atrial fibrillation associated with subclinical hyperthyroidism is limited, and few studies have described the risk in patients with high normal thyroid function and hypothyroidism.

\section{What this study adds}

Patients with subclinical hyperthyroidism had a $30 \%$ increased risk of atrial fibrillation compared with euthyroid patients, and patients with high normal thyroid function had a $12 \%$ increased risk of atrial fibrillation

Both overt and subclinical hypothyroidism were associated with a lower risk of atrial fibrillation compared with euthyroid patients The study suggests that there should be an increased focus on atrial fibrillation in patients with subclinical hyperthyroidism and high normal thyroid function.

34 Sgarbi JA, Villaça FG, Garbeline B, Villar HE, Romaldini JH. The effects of early antithyroid therapy for endogenous subclinical hyperthyroidism in clinical and heart abnormalities. $J$ Clin Endocrinol Metab 2003;88:1672-7.

35 Zhou Z-H, Ma L-L, Wang L-X. Risk factors for persistent atrial fibrillation following successful hyperthyroidism treatment with radioiodine therapy. Intern Med 2011:50:2947-51.

36 Cooper DS. Clinical practice. Subclinical hypothyroidism. N Engl J Med 2001;345:260-5

37 Biondi B, Kahaly GJ. Cardiovascular involvement in patients with different causes of hyperthyroidism. Nat Rev Endocrinol 2010;6:431-43.

38 Frost L, Andersen LV, Vestergaard P, Husted S, Mortensen LS. Trend in mortality after stroke with atrial fibrillation. Am J Med 2007;120:47-53.

39 Mukamal KJ, Tolstrup JS, Friberg J, Jensen G, Grønbaek M. Alcohol consumption and risk of atrial fibrillation in men and women: the Copenhagen City Heart Study. Circulation 2005;112:1736-42.
40 Frost L, Vestergaard P. Alcohol and risk of atrial fibrillation or flutter: a cohort study. Arch Intern Med 2004;164:1993-8.

\section{Accepted: 05 November 2012}

\section{Cite this as: BMJ 2012;345:e7895}

This is an open-access article distributed under the terms of the Creative Commons Attribution Non-commercial License, which permits use, distribution, and reproduction in any medium, provided the original work is properly cited, the use is non commercial and is otherwise in compliance with the license. See: $\mathrm{http}: / /$ creativecommons.org/licenses/by$\mathrm{nc} / 2.0 /$ and http://creativecommons.org/licenses/by-nc/2.0/legalcode. 


\section{Tables}

Table 1/ Definitions of thyroid disease and thyroid dysfunction levels used in study

Thyroid stimulating hormone ( $\mathrm{mlU} / \mathrm{L})$ Free thyroxine (pmol/L) Total thyroxine ( $\mathrm{mmol} / \mathrm{L})$

Thyroid dysfunction definitions

\begin{tabular}{lccc}
\hline Euthyroidism & $0.2-5.0$ & $9-22$ & $<$ \\
\hline Overt hypothyroidism & $>5.0$ & $<0-140$ & $<60$ \\
\hline Subclinical hypothyroidism & $>5.0$ & $9-22$ & $>140$ \\
\hline Overt hyperthyroidism & $<0.2$ & $>22$ & $60-140$ \\
\hline Subclinical hyperthyroidism & $<0.2$ & $9-22$ & $60-140$ \\
\hline Thyroid stimulating hormone (TSH) level dependent thyroid dysfunction definitions & $9.4-5.0$ & $9-22$ & $60-140$ \\
\hline Euthyroidism & $0.2-0.4$ & $9-22$ & $60-140$ \\
\hline High normal euthyroidism & $0.1-0.2$ & $9-22$ & $60-140$ \\
\hline Subclinical hyperthyroidism (reduced TSH) & $<0.1$ & $9-22$ & 6 \\
\hline
\end{tabular}




\begin{tabular}{|c|c|c|c|c|c|c|}
\hline \multirow[b]{2}{*}{ Characteristic } & \multicolumn{5}{|c|}{ Thyroid status } & \multirow[b]{2}{*}{$\begin{array}{c}\text { Total population } \\
(n=586460)\end{array}$} \\
\hline & $\begin{array}{c}\text { Overt } \\
\text { hypothyroidism } \\
(n=1670)\end{array}$ & $\begin{array}{l}\text { Subclinical } \\
\text { hypothyroidism } \\
(\mathrm{n}=12 \text { 087) }\end{array}$ & $\begin{array}{l}\text { Euthyroidism } \\
(\mathrm{n}=562 \text { 461) }\end{array}$ & $\begin{array}{c}\text { Subclinical } \\
\text { hyperthyroidism } \\
(n=6276)\end{array}$ & $\begin{array}{c}\text { Overt } \\
\text { hyperthyroidism } \\
(\mathrm{n}=3966)\end{array}$ & \\
\hline Mean (SD) age (years): & $53.7(18.1)$ & $54.5(18.5)$ & $48.6(18.2)$ & $60.6(19.4)$ & $51.8(19.4)$ & $48.9(18.3)$ \\
\hline Women & $53.2(18.4)$ & $54.7(18.6)$ & $47.7(19.0)$ & $60.3(20.1)$ & $51.6(19.8)$ & $48.1(19.1)$ \\
\hline Men & $55.6(16.6)$ & $53.5(18.1)$ & $50.0(16.8)$ & $61.5(16.8)$ & $52.8(17.4)$ & $50.2(16.9)$ \\
\hline \multicolumn{7}{|l|}{ Sex: } \\
\hline Women & $1355(81)$ & $9675(80)$ & $336520(60)$ & $4784(76)$ & $3171(80)$ & 355505 (61) \\
\hline Men & $315(19)$ & $2412(20)$ & $225941(40)$ & $1492(24)$ & $795(20)$ & 230955 (39) \\
\hline \multicolumn{7}{|l|}{$\begin{array}{l}\text { Mean (SD) thyroid } \\
\text { function: }\end{array}$} \\
\hline $\mathrm{TSH}(\mathrm{mlU} / \mathrm{L})$ & $63.77(44.64)$ & $9.24(7.24)$ & $1.56(0.84)$ & $0.086(0.061)$ & $0.038(0.043)$ & $1.871(4.428)$ \\
\hline FT4 (pmol/L) & $6.28(1.93)$ & $13.11(2.18)$ & $15.05(2.30)$ & $16.89(2.62)$ & $42.61(22.28)$ & $15.94(8.27)$ \\
\hline \multicolumn{7}{|l|}{ Comorbidity: } \\
\hline $\begin{array}{l}\text { Peripheral vascular } \\
\text { disease }\end{array}$ & $3(0)$ & $19(0)$ & $676(0)$ & $17(0)$ & $10(0)$ & $725(0)$ \\
\hline $\begin{array}{l}\text { Cerebral vascular } \\
\text { disease }\end{array}$ & $6(0)$ & $78(1)$ & $3024(1)$ & $57(1)$ & $25(1)$ & $3190(1)$ \\
\hline Ischaemic heart disease & $9(1)$ & $59(0)$ & $2652(0)$ & $61(1)$ & $19(0)$ & $2800(0)$ \\
\hline Malignancy & $22(1)$ & $72(1)$ & $2552(0)$ & $61(1)$ & $26(1)$ & $2733(0)$ \\
\hline COPD & $8(0)$ & $48(0)$ & $1930(0)$ & $61(1)$ & $26(1)$ & $2073(0)$ \\
\hline Diabetes & $6(0)$ & $39(0)$ & $2381(0)$ & $47(1)$ & $19(0)$ & $2492(0)$ \\
\hline Rheumatic disease & $1(0)$ & $9(0)$ & $363(0)$ & $13(0)$ & $4(0)$ & $390(0)$ \\
\hline Chronic renal failure & $2(0)$ & $7(0)$ & $224(0)$ & $6(0)$ & $1(0)$ & $240(0)$ \\
\hline Gastric ulcer & $3(0)$ & $22(0)$ & $1187(0)$ & $25(0)$ & $16(0)$ & $1253(0)$ \\
\hline Congestive heart failure & $6(0)$ & $35(0)$ & $997(0)$ & $32(1)$ & $11(0)$ & $1081(0)$ \\
\hline \multicolumn{7}{|l|}{$\begin{array}{l}\text { Charlson comorbidity } \\
\text { index: }\end{array}$} \\
\hline 0 & $1629(97)$ & $11929(98)$ & $556268(98)$ & $6104(96)$ & $3930(97)$ & $579860(98)$ \\
\hline 1 & $24(1)$ & $179(1)$ & $7418(1)$ & $157(2)$ & $73(2)$ & $7842(1)$ \\
\hline 2 & $23(1)$ & $89(1)$ & $3037(1)$ & $78(1)$ & $28(1)$ & $3255(1)$ \\
\hline$\geq 3$ & $6(0)$ & $38(0)$ & $1669(0)$ & $34(0)$ & $19(0)$ & $1766(0)$ \\
\hline \multicolumn{7}{|l|}{$\begin{array}{l}\text { Yearly family income, by } \\
\text { quintiles: }\end{array}$} \\
\hline 0 & $234(14)$ & $2096(18)$ & $103987(19)$ & $1320(21)$ & $732(19)$ & $108369(19)$ \\
\hline 1 & $384(23)$ & $2741(23)$ & $107427(19)$ & $1703(28)$ & $930(24)$ & $113185(20)$ \\
\hline 2 & $357(22)$ & $2467(21)$ & $111809(20)$ & $1267(21)$ & $799(21)$ & $116669(20)$ \\
\hline 3 & $352(21)$ & $2336(20)$ & $114707(21)$ & $1027(17)$ & $765(20)$ & $119187(21)$ \\
\hline 4 & $321(19)$ & $2230(19)$ & $115735(21)$ & $833(14)$ & $662(17)$ & $119781(21)$ \\
\hline
\end{tabular}

$\mathrm{TSH}=$ thyroid stimulating hormone, COPD=chronic obstructive pulmonary disease. 


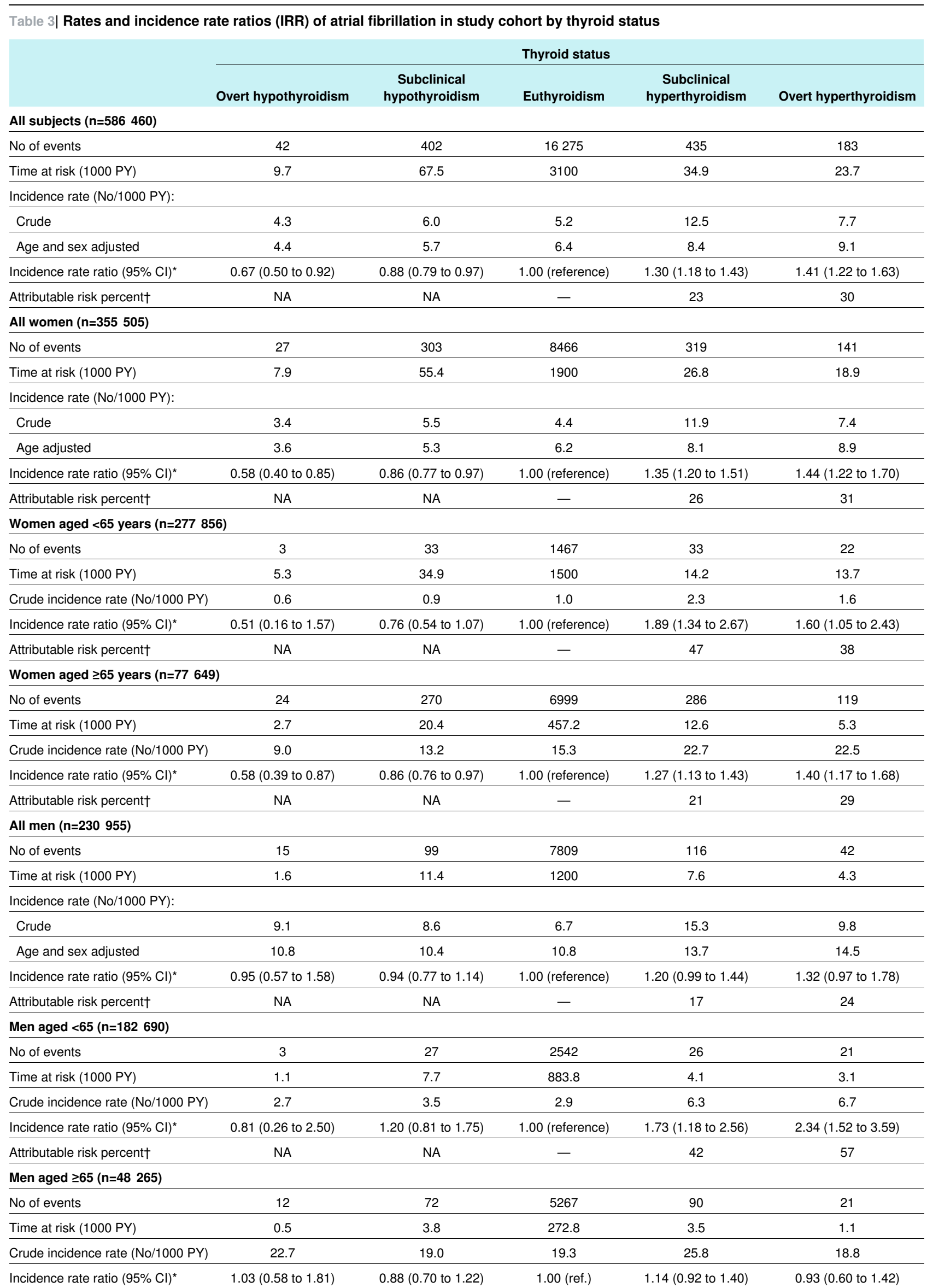


Table 3 (continued)

\begin{tabular}{lcccc} 
& \multicolumn{2}{c}{ Thyroid status } \\
\cline { 2 - 5 } & Overt hypothyroidism & $\begin{array}{c}\text { Subclinical } \\
\text { hypothyroidism }\end{array}$ & Euthyroidism & $\begin{array}{c}\text { Subclinical } \\
\text { hyperthyroidism }\end{array}$ \\
Attributable risk percent† & NA & NA & - & 12 \\
\hline
\end{tabular}

$P Y=$ person years. $N A=$ not applicable.

*Risk estimates are adjusted for sex, age, calendar year, Charlson comorbidity index, and socioeconomic status. †Attributable risk percent $=(\mathrm{IRR}-1) / \mathrm{IRR} \times 100$ 
Table 4| Rates and incidence rate ratios (IRR) of atrial fibrillation in study cohort by level of thyroid stimulating hormone (TSH) with normal free thyroxine concentration

\begin{tabular}{|c|c|c|c|c|}
\hline & \multicolumn{2}{|c|}{ Euthyroidism } & \multicolumn{2}{|c|}{ Subclinical hyperthyroidism } \\
\hline & Normal & High normal & Reduced TSH & Suppressed TSH \\
\hline TSH level (mIU/L) & $0.4-5.0$ & $0.2-0.4$ & $0.1-0.2$ & $<0.1$ \\
\hline \multicolumn{5}{|l|}{ Rate of atrial fibrillation } \\
\hline No of events & 15652 & 623 & 155 & 280 \\
\hline Time at risk (1000 PY) & 3000.0 & 73.9 & 13.8 & 21.1 \\
\hline \multicolumn{5}{|l|}{ Incidence rate (No/1000 PY): } \\
\hline Crude & 4.9 & 8.4 & 11.2 & 13.3 \\
\hline Age adjusted & 8.0 & 8.8 & 8.9 & 10.3 \\
\hline Incidence rate ratio $(95 \% \mathrm{Cl})^{*}$ & 1.00 (reference) & $1.12(1.03$ to 1.21$)$ & $1.16(0.99$ to 1.36$)$ & $1.41(1.25$ to 1.59$)$ \\
\hline Attributable risk percent† & & 11 & 14 & 29 \\
\hline
\end{tabular}

$\mathrm{PY}=$ person years. $\mathrm{NA}=$ not applicable.

${ }^{*}$ Risk estimates are adjusted for sex, age, calendar year, Charlson comorbidity index, and socioeconomic status.

$\dagger$ Attributable risk percent $=(\operatorname{IRR}-1) / \operatorname{IRR} \times 100$. 


\section{Figures}

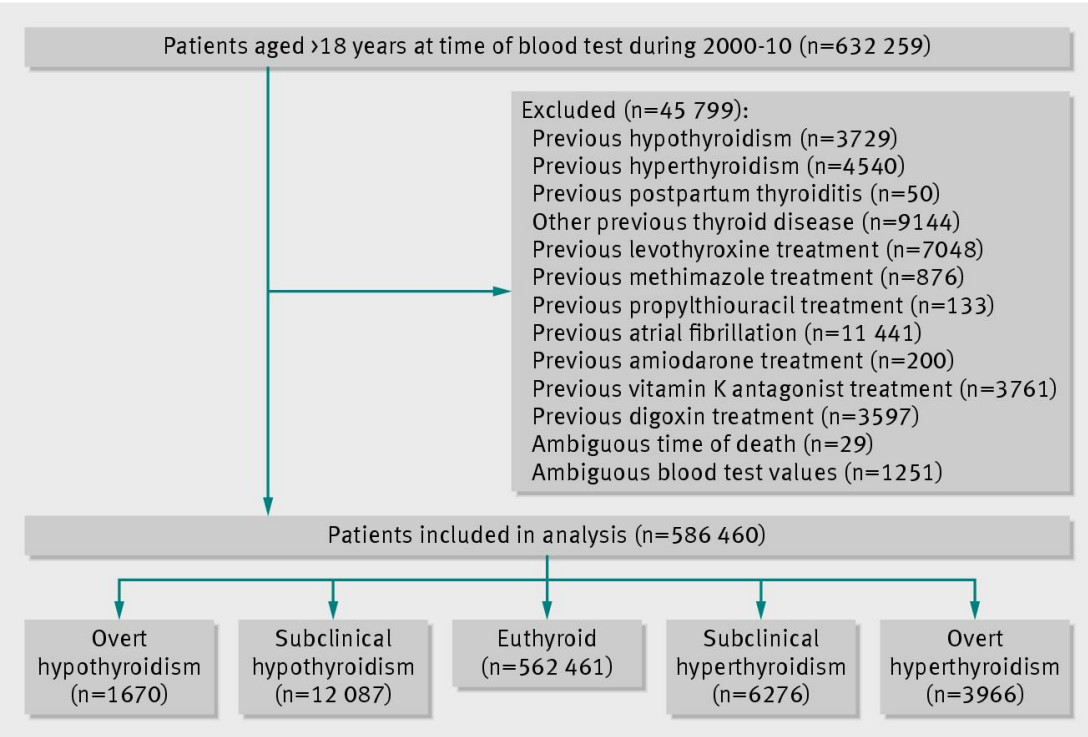

Fig 1 Flowchart of study cohort selection.

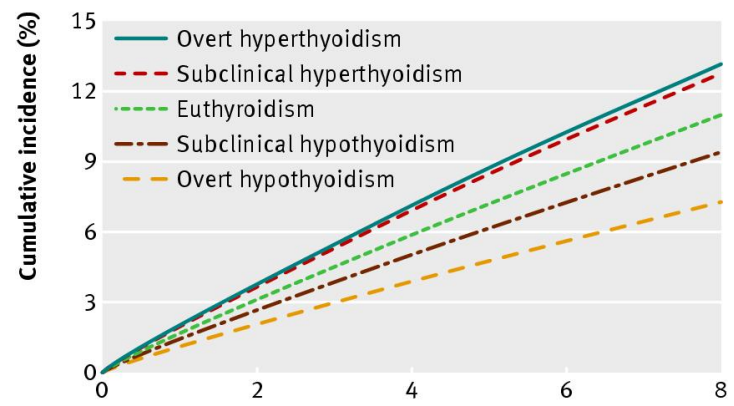

Follow-up time (years)

Fig 2 Cumulative incidence of atrial fibrillation in relation to thyroid dysfunction at baseline thyroid screening (age >65 years). Values adjusted for competing risk of death from all causes

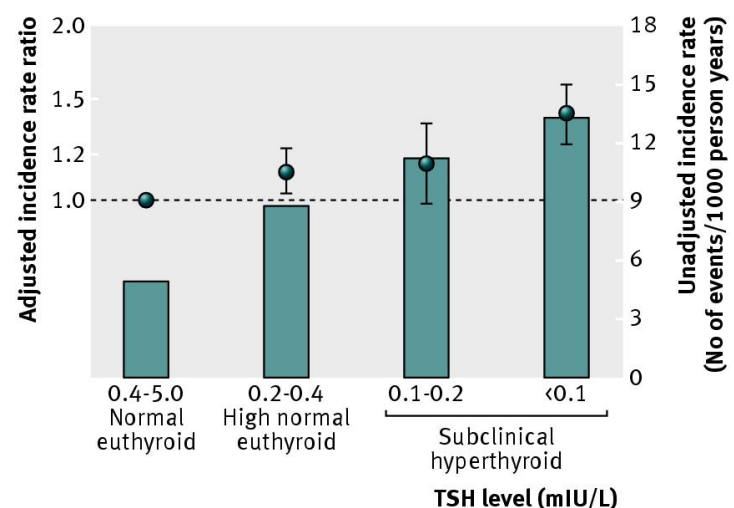

Fig 3 Adjusted incidence rate ratios (box and whiskers) and unadjusted incidence rates per 1000 person years (bars) for atrial fibrillation by level of thyroid stimulating hormone (TSH) in euthyroid and subclinical hyperthyroid patients in study cohort 